Instructions for authors, subscriptions and further details:

http://ijrs.hipatiapress.com

\title{
"Deseo trabajo estable y buen sueldo". Historias de vida sobre la continuidad educativa de la población gitana cántabra
}

Carlos Hernández-Borja ${ }^{1}$ and Carmen Álvarez-Álvarez²

1) Fundación Diagrama, Spain

2) University of Cantabria, Spain

Date of publication: March $15^{\text {th }}, 2021$

Edition period: March 2021 - July 2021

To cite this article: Hernández-Borja, C., \& Álvarez-Álvarez, C. (2021). "Deseo trabajo estable y buen sueldo". Historias de vida sobre la continuidad educativa de la población gitana cántabra. International Journal of Roma Studies, 3(1), 24-45. doi: 10.17583/ijrs.2021.6987

To link this article: http://dx.doi.org/10.17583/ijrs.2021.6987

PLEASE SCROLL DOWN FOR ARTICLE

The terms and conditions of use are related to the Open Journal System and to Creative Commons Attribution License (CCAL). 


\section{"I want a stable job and good salary". Life stories about the educational continuity of the Cantabrian Roma/Gitano population}

Carlos Hernández-Borja Roma mediator. Fundación Diagrama

\author{
Carmen Álvarez-Álvarez \\ University of Cantabria
}

\section{Abstract}

The educational continuity of the Roma population can influence its socio-cultural improvement; however, it is necessary to continue researching the reasons that contribute most to it. In this work we ask ourselves why some Roma continue studying and what factors have contributed and hindered this decision, developing the field work in a community where there are no previous studies, Cantabria. We have contacted 12 people from the Roma/Gitano community and listened to their life stories, following a communicative methodology. The results show twelve factors that hinder educational continuity, such as the racism that exists in the school, the memorisation as usual academic form and the fear of failure. We have also identified twelve factors that favour educational continuity of the Roma people, highlighting personal determination to study, pride in pursuing a professional goal and achieving it, or the current situation of the labour market and the crisis. It is possible to conclude that the Romany population is in a phase of transition between tradition and modernity and that there is a high probability that the new generations will opt for their educational continuity.

Keywords: Roma People, Gitano, ethnic minority, educational continuity, life stories. 


\section{"Deseo trabajo estable y buen sueldo". Historias de vida sobre la continuidad educativa de la población gitana cántabra}

Carlos Hernández-Borja Mediador gitano. Fundación Diagrama

\author{
Carmen Álvarez-Álvarez \\ Universidad de Cantabria
}

\section{Resumen}

La continuidad educativa de la población gitana puede influir en su mejora sociocultural, sin embargo, es necesario continuar investigando sobre las razones que más contribuyen a la misma. En este trabajo nos preguntamos por qué algunas personas gitanas continúan estudiando y qué factores han contribuido y entorpecido esta decisión, desarrollando el trabajo de campo en una comunidad en la que no hay estudios previos, Cantabria. Hemos contactado con 12 personas de la comunidad gitana y hemos escuchado sus historias de vida, siguiendo una metodología comunicativa. Los resultados muestran doce factores que dificultan la continuidad educativa como el racismo existente en la escuela, las formas académicas memorísticas y el temor al fracaso. También hemos identificado doce factores que favorecen la continuidad educativa del pueblo gitano, destacando la determinación personal a estudiar, el orgullo de perseguir con ahínco y lograr una meta profesional o la situación actual del mercado laboral y la crisis. Es posible concluir que el pueblo gitano está en una fase de tránsito entre tradición y modernidad y que hay una alta probabilidad de que las nuevas generaciones apuesten por su continuidad educativa.

Palabras clave: población gitana, población romaní, minoría étnica, continuidad educativa, historias de vida.

2021 Hipatia Press

ISSN: $2462-425 \mathrm{X}$

DOI: $10.17583 / \mathrm{ijrs} .2021 .6187$

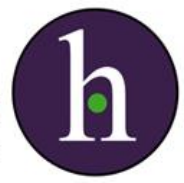




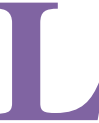

a comunidad gitana constituye la minoría étnica más numerosa y antigua de Europa (Carmona Santiago et al., 2019) y aunque tiene una identidad cultural propia ha venido resistiendo durante siglos todo tipo de persecuciones, intentos de asimilación, y hasta de aniquilación (Lorenzo Moledo et al., 2020). En Europa (y España) está arraigado un anti gitanismo: la cultura gitana está silenciada y es desconocida por el común de la población, el pueblo gitano constituye una minoría aislada y predominan los estereotipos (Díez-Palomar et al., 2018; Marques Gonçalves, 2020; Rostas \& Kostka, 2014), produciendo tensiones y choques culturales en las comunidades educativas de los centros escolares (Abajo \& Carrasco, 2004; Dimitrova, Ferrer-Wreder, et al., 2018; Lorenzo Moledo et al., 2020).

La continuidad educativa de la población gitana, cómo lograr su éxito escolar y disminuir su fracaso o abandono, sigue siendo objeto de investigaciones recientes a nivel nacional e internacional (Alexiadou, 2019; Curcic et al., 2018; Díez-Palomar et al., 2018; Dimitrova, Ferrer-Wreder, et al., 2018; Dimitrova, Johnson, et al., 2018; Goenechea \& Gallego, 2020; Helakorpi et al., 2019; Lorenzo Moledo et al., 2020; Rostas \& Kostka, 2014). Sin embargo, la educación del pueblo gitano sigue siendo un asunto poco investigado, la mayor parte de los estudios se han centrado en la exclusión y no han predominado los estudios que den voz al pueblo gitano y contribuyan a incrementar su éxito educativo para contribuir a mejorar su situación en nuestro país (Abajo \& Carrasco, 2004; Goenechea \& Gallego, 2020; Lorenzo Moledo et al., 2020). Si bien a nivel internacional es frecuente el término de roma, en España el término predominante es el de gitano, motivo por el cual lo empleamos, como en estudios previos (Goenechea \& Gallego, 2020).

Tanto en el panorama nacional como internacional se han tomado medidas para garantizar la inclusión y la educación de las personas gitanas en igualdad, y se observan algunos progresos, pero aún se debe trabajar profusamente por la escolarización y el éxito educativo de la infancia gitana (Abajo \& Carrasco, 2004; Alexiadou, 2019). Con demasiada frecuencia se ha normalizado el bajo rendimiento escolar de la población gitana y el abandono temprano (Curcic et al., 2018; Díez-Palomar et al., 2018), y no siempre se ha contemplado la existencia de profundas diferencias entre territorios, no existiendo una homogeneidad educativa entre la comunidad gitana (Abajo \& Carrasco, 2004; Rostas \& Kostka, 2014). Queda mucho por 
hacer para lograr el éxito del alumnado gitano y esta investigación pretende contribuir a ello.

La escolarización del alumnado gitano en la Educación Primaria en España prácticamente se ha logrado (Lorenzo Moledo et al., 2020), pero en la Educación Secundaria es reducida y presenta unos índices de absentismo y/o abandono escolar elevados, siendo muy escasos los estudiantes que concluyen Formación Profesional o una titulación universitaria (Abajo \& Carrasco, 2004; Goenechea \& Gallego, 2020), algo que también sucede en otros países europeos (Curcic et al., 2018). La evaluación del reciente Programa nacional Promociona, dirigido a promover la continuidad educativa del alumnado en la Educación Primaria y en la Secundaria Obligatoria (ESO) arrojó unos resultados positivos, demostrando que la probabilidad de obtener éxito escolar resultó 3,22 veces mayor en Promociona que en el grupo control. Los factores determinantes del éxito escolar del programa Promociona fueron el personal, la ayuda extraescolar y las tutorías (Aguilar Jurado et al., 2020).

Un estudio reciente ofrece algunos datos relevantes (Goenechea \& Gallego, 2020): (1) La proporción de personas gitanas con estudios superiores en España ha pasado del 1,1\% en 2005 al 3,2\% en 2018. (2) Sin embargo, la diferencia es muy significativa al comparar el porcentaje con el del conjunto de la población española con estudios superiores, que ronda el $35 \%$ y, muy especialmente, al comparar regiones. (3) En la Universidad de Cádiz el porcentaje de personas gitanas es de apenas el 2\%. En otras universidades, como la de Cantabria, ese porcentaje es inferior al $0,05 \%$ (según datos inéditos facilitados por las asociaciones gitanas). La realidad regional es poco homogénea y cada área tiene sus propias peculiaridades.

A partir de una investigación desarrollada en Croacia con niñas y niños gitanos de Educación Primaria con el objetivo de potenciar prácticas educativas exitosas (Curcic et al., 2018) se consideró prioritario eliminar los prejuicios de los maestros, para lo que examinaron el patrimonio cultural gitano y crearon comunidades de colaboración dentro y fuera de las aulas. Otro estudio desarrollado en Bulgaria comparando las percepciones y aspiraciones educativas de jóvenes gitanos, sus familias y profesorado demostró que el clima escolar influye en las expectativas de la comunidad gitana y que no deben desatenderse las dimensiones actitudinales para promover el éxito escolar (Dimitrova, Ferrer-Wreder, et al., 2018). 
Asimismo, otra investigación en Hungría puso de relieve el negativo efecto de las prácticas educativas segregadoras, porque contribuyen a la discriminación y demanda estrategias políticas inclusivas ambiciosas (Rostas \& Kostka, 2014). La investigación realizada con once personas gitanas que han tenido éxito en la educación de Eslovaquia, Hungría, Rumania, Serbia y España concluye que las trayectorias educativas no están predeterminadas, sino que son negociadas y moldeadas por los participantes en sus interacciones con la escuela, la familia y las redes sociales más amplias, y están enmarcadas por las prácticas, recursos y políticas de bienestar en torno a la igualdad y las oportunidades, a las que critica de retóricas, al fallar tanto a nivel local como nacional (Alexiadou, 2019). Esta investigación valora el apoyo de las ONGs que proveen de recursos, financiación, servicios y fundamentos legales a familias y centros, en tanto que son aliados en este proceso de mejora de la calidad educativa. Por otro lado, otro estudio en Rumanía ha puesto de manifiesto las dificultades que encuentran las personas gitanas (las formadas y no formadas) en encontrar trabajo en ámbitos no tradicionales debido a que la gran mayoría de los empresarios son reacios a integrar a personas gitanas en el mercado laboral (Mihaela \& Luminița, 2020).

En España en 2004 se realizó un estudio en cinco comunidades autónomas (Abajo \& Carrasco, 2004), verificándose que se daban una gran diversidad de trayectorias de continuidad educativa entre la población gitana. Los diez factores que, por orden de importancia, los participantes en el estudio más han destacado, han sido: (1) sentirse valorado y apoyado: la autoestima unida a la aceptación por parte de otras personas. (2) El apoyo de su madre y/o padre en el sentido del proyecto y en sentido de la ayuda. (3) El fuerte convencimiento individual para proseguir sus estudios. (4) El propio coraje, esfuerzo y capacidad para afrontar y buscar soluciones para superar las barreras que le imponían a la persona. (5) El fenotipo y su modo de autopresentación (vestimenta, peinado, manera de hablar y relacionarse, etc.) no corresponde al aspecto que tradicionalmente se asocia a la apariencia gitana. (6) Un apoyo desde el profesorado y el centro educativo si no constante, sí en los momentos de toma de decisiones que pueden afectar la continuidad educativa. (7) La percepción de un sentimiento de igualdad con la sociedad mayoritaria. (8) El acceso a becas, especialmente para aquellos que han tenido trayectorias académicas más largas. (9) La necesidad de la 
persona por salir de un entorno de precariedad económica, que se espera superar con la obtención de una titulación académica y una mejor inserción laboral. (10) Su actitud abierta e independiente.

Otra investigación de 2018, sobre las características que han contribuido al éxito en el aprendizaje de las matemáticas por parte de estudiantes gitanos puso de manifiesto algunos factores relevantes, no coincidentes con los estudios previos (Díez-Palomar et al., 2018): (1) desarrollar una identidad académica basada en valores como el esfuerzo, el atractivo de la escolarización, y la motivación para el aprendizaje; (2) la importancia del papel del maestro en la creación de actividades curriculares desafiantes, aumentando la oportunidades de aprendizaje, basadas en altas expectativas y no en estereotipos sociales, y evitar prácticas negativas como la agrupación de capacidades; (3) el papel de la familia en la búsqueda de apoyo y el empoderamiento de los estudiantes gitanos sobre su capacidad para tener éxito en la escuela; (4) la interacción entre pares basada en la valoración de la escolaridad, estableciendo una "red" de apoyo (trabajo en grupo) basado en la solidaridad e incluso generando cadenas de solidaridad (estudiantes ayudando a otros estudiantes); y (5) utilizando estrategias "tradicionales" como el resto de los estudiantes (estudiar para aprobar el exámenes, resolver las actividades del aula, hacer los deberes asignados, etc.).

En 2018 se realizó otro estudio en la Comunidad Valenciana sobre el abandono escolar temprano entre los jóvenes de etnia gitana entre 12 y 15 años y los factores que más les influyeron para no continuar estudiando fueron: el racismo y la discriminación percibida, la desmotivación por seguir estudiando y la idea de seguir con los roles familiares tradicionales (Bitrián Ferrer \& Agost Felip, 2018).

Ante estos resultados, nos planteamos: ¿serán los mismos ahora en Cantabria teniendo en cuenta las diferencias existentes entre regiones, la crisis que atravesamos y que han pasado más de quince años desde uno de los estudios nacionales más amplios (Abajo \& Carrasco, 2004)? La población de Cantabria no formó parte de la muestra de ninguno de los estudios que hemos examinado. Por todo ello, en este artículo presentamos una investigación en esta comunidad que sirva de punto de inicio de la investigación al respecto: que muestre la realidad a través de los ojos de las personas gitanas que han apostado por su continuidad educativa y ofrezca claves para orientar futuras acciones de mejora. 


\section{Método}

Nuestro objetivo principal es realizar un estudio exploratorio para conocer cuáles son los factores que más han contribuido a que haya continuidad educativa por parte de la población gitana en Cantabria e identificar en cuáles hace falta incidir más, a partir del análisis de testimonios de personas con perspectivas de continuidad escolar.

En línea con este planteamiento, hemos seguido las pautas de la metodología comunicativa (Vargas et al., 2004), siendo el método de investigación el estudio de casos (Curcic et al., 2018; Díez-Palomar et al., 2018) y el modelo de investigación el narrativo-biográfico (Cantón-Delgado, 2018; O'Nions, 2015), ya que hemos encontrado algunos antecedentes de estudios que emplean métodos cualitativos, de corte narrativo-biográfico, etnográfico o mediante entrevistas para abordar la continuidad educativa de la población gitana en los que nos hemos apoyado (Abajo \& Carrasco, 2004; Baracsi, 2016; Goenechea \& Gallego, 2020; Helakorpi et al., 2019).

En concreto, en este estudio nos hemos decantado por la realización de historias de vida, demandando relatos biográficos a diferentes personas gitanas que han alcanzado algún grado formativo o lo están tratando de alcanzar. La selección de los participantes fue realizada por un miembro de su comunidad (autor1), conocedor de la trayectoria escolar de los mismos, tras trabajar para una asociación promotora de la educación para la minoría gitana. Los dos autores firmantes del artículo se coordinaron para elaborar un guion básico de posibles preguntas a realizar en los relatos autobiográficos y para establecer los criterios a seguir en la selección de los participantes: deseábamos contar con personas de edades comprendidas entre los 15 y los 45 años, que fuesen hombres y mujeres y que tuviesen diferentes experiencias educativas (ESO, Bachillerato, Ciclos formativos de grado medio, Ciclos formativos de grado superior, estudios universitarios y personas que hubiesen retomado los estudios de adultos). El autor1 estableció su propia cronología para compatibilizar su vida laboral y familiar y realizar las historias de vida a lo largo de 2019.

Los relatos autobiográficos se realizaron por separado con cada uno de los participantes por parte del autor1. Finalmente se desarrollaron a lo largo del año 2019 y 2020 a través de contactos cara a cara en espacios acogedores para ambos. Estaba previsto realizar todas las historias de vida en 2019, pero 
dado que solo contábamos con 8 colaboraciones (6 hombres y 2 mujeres), para facilitar la colaboración de más personas decidimos dedicar seis meses más a contactar con las personas implicadas, especialmente mujeres. Así, logramos la participación de 4 personas más. Este reajuste de la planificación inicial se vio alterado por la pandemia por COVID-19, realizándose la última de estas cuatro en septiembre de 2020. Estas cuatro últimas entrevistas nos permitieron alcanzar el reto inicial de contar con personas de edades comprendidas entre los 16 y los 40 años, que fuesen hombres y mujeres, y que tuviesen diferentes experiencias educativas.

Las personas fueron informadas del objetivo del estudio y firmaron un consentimiento informado en papel previamente al inicio de la grabación donde se recogían los compromisos a asumir por parte de los colaboradores (participación voluntaria y revisión de trascripciones) y de los investigadores (tratamiento confidencial, desinteresado e imparcial de los datos, no difusión de las trascripciones, anonimato de los participantes). Los participantes que han colaborado han sido:

1. Abel. 16 años. Cursa segundo de ESO.

2. Álex. 19 años. Cursa Bachillerato.

3. Miguel. 21 años. Cursa ciclo formativo de grado medio.

4. Adrián. 23 años. Cursa ciclo formativo de grado medio.

5. Belén. 26 años. Concluyendo un grado universitario. Cursa ciclo formativo degrado superior online.

6. Jhony. 27 años. Concluyó grado universitario.

7. Brian. 28 años. Cursa ciclo formativo de grado superior.

8. Manuel. 29 años. Cursa un grado universitario

9. Moisés. 31 años. Abandonó en tercero de ESO.

10.Roberto. 39 años. Concluyó la ESO de adulto. Cursa certificado profesionalidad de grado superior.

11.Richy. 40 años. Concluyó la ESO de adulto.

12.Sandra. 42 años. Concluyó la ESO de adulta.

Las historias de vida versaron sobre tres ejes:

(1) Trayectoria escolar. Se formularon preguntas como: ¿Qué razones tienes para estudiar/ no hacerlo? ¿Qué es lo que más/menos te ha motivado a estudiar en tu vida? ¿Conoces a gitanos que estudian? ¿Sueles hablar con otros gitanos sobre tu experiencia escolar, qué os contáis? ¿Algo de la 
escuela te ha emocionado alguna vez? ¿Te ha marcado algún docente en positivo/negativo? ¿Qué es lo que más recuerdas de tus diferentes etapas educativas? ¿Alguna vez te has puesto a aprender o estudiar algo por voluntad propia?

(2) Influencias en el estudio. Se formularon preguntas como: ¿Qué opinión te influye más: la de tus padres, la de tus profesores, la del pastor, otras? ¿Tus padres/profesores/pastores/otros te han animado a estudiar? ¿En tu familia alguien te apoyó para estudiar? ¿Qué te gustaría haber estudiado y no has hecho y por qué? ¿Por qué te dedicas a lo que te dedicas? ¿Tu vida familiar impide o dificulta estudiar? ¿Si pudieses dar vuelta atrás estudiarías?

(3) Futuro educativo del pueblo gitano. Se formularon preguntas como: ¿En el futuro el pueblo gitano estudiará más que ahora? ¿Cómo lo ves? ¿Te gustaría que tus hijos estudien, por qué? ¿Qué hay que hacer para que el pueblo gitano deje de fracasar en la escuela? ¿Qué te gustaría decir, pedir o reivindicar, tanto a la comunidad gitana como a la paya en materia de Educación? Si tuvieras el poder político para cambiar el sistema educativo en beneficio de la comunidad gitana ¿qué harías?

Todos los datos con los que se ha trabajado se grabaron en audio (autor1) porque interesaba recoger sus palabras con la máxima literalidad posible, no siendo posible, por tanto, analizar la comunicación no verbal. Las historias de vida fueron transcritas por parte del autor1. Una vez recopilada toda la información se procedió a organizar y analizar las afirmaciones realizadas por los participantes mediante un proceso de análisis de contenido por parte del autor2, con la intención de identificar evidencias sobre el objeto de estudio. No obstante, la comunicación entre los autores en todo momento ha sido fluida. Para organizar los resultados se crearon tablas empleando herramientas de Microsoft Office (Word y Excel). Antes de realizar el análisis preveíamos que contaríamos con datos sobre factores facilitadores y entorpecedores y que en ese sentido se deberían organizar los datos.

El análisis se realizó de forma manual, no empleándose ningún software específico, siendo la unidad de análisis básica la palabra (ejemplo: prejuicio, soledad, apoyo...). Los datos, como supusimos, obedecían a dos grandes categorías: (1) factores que dificultaban la continuidad educativa y (2) factores que facilitaban la continuidad educativa de las personas gitanas. Dado que muchas respuestas se repetían, tanto en los factores 
entorpecedores como en los facilitadores, cuando ordenamos los resultados decidimos recoger en primer lugar aquellos que suscitaban un mayor acuerdo entre las personas participantes. Así, durante el propio análisis, emergieron 12 factores entorpecedores y 12 factores facilitadores que alcanzaron diferentes grados de consenso. Todos los factores recogidos en el artículo han sido señalados por al menos 3 informantes, sin señalar el número de menciones obtenidas porque el foco del estudio no es realizar un análisis estadístico sobre los testimonios recogidos, sino construir una narrativa. Sin embargo, cabe destacar, por su alta coincidencia, que el mayor consenso fue el logrado por el primer factor entorpecedor (racismo vivido en la escuela, señalado por 10 de las personas consultadas), seguido a gran distancia del resto, dado que casi todos los factores recogidos son señalados por un número de personas semejante (entre 3 y 6 , en la mayor parte de los casos).

El hecho de contar con un guion de entrevista articulado en tres ejes ha permitido triangular los datos tras su recogida y ha facilitado encontrar semejanzas y diferencias entre los testimonios de los participantes. No obstante, además, la redacción de los resultados se dio a leer a tres miembros de la comunidad gitana para triangularlos (autor1) y se les pidió que los evaluasen y realizasen propuestas de cambios. Cada una de las tres personas gitanas consultadas lo leyó por separado, no siendo conocedores de que también se darían a revisar a otros dos participantes más. Las tres personas gitanas lo revisaron y coincidieron en que los resultados constituyen una síntesis realista y actual de los factores entorpecedores y facilitadores que están afectando a la comunidad gitana de Cantabria en estos momentos, dando su visto bueno a los factores identificados en el análisis de datos y proponiendo uno de ellos pequeños cambios de redacción que fueron tenidos en cuenta.

Se han respetado los principios éticos básicos como la preservación del anonimato (los nombres empleados para elaborar este artículo son pseudónimos), la revisión de las trascripciones por parte de los interesados y del análisis de datos realizado, colaboración anónima y desinteresada e imparcialidad en el tratamiento de los datos. 


\section{Resultados}

Nuestro objetivo es identificar los factores que más han contribuido a que haya continuidad educativa por parte de la población gitana en Cantabria e identificar en cuáles hace falta incidir más. Hemos organizado los datos obtenidos en torno a dos grandes categorías emergentes: (1) factores que dificultan la continuidad educativa, (2) factores que facilitan la continuidad educativa de las personas gitanas participantes. En cada una de estas categorías ofreceremos las diferentes ideas que los mismos nos han compartido, incorporando algún testimonio ilustrativo.

\section{Factores que dificultan la continuidad educativa.}

Son muchos los factores identificados en las historias de vida que dificultan, y en ocasiones desmoralizan, a las personas gitanas a continuar estudiando. En síntesis, recogemos doce:

El factor más señalado es el (1) racismo existente en la escuela. Aunque algunos no lo han experimentado o lo han hecho con diferente intensidad en las diferentes etapas educativas que han atravesado, la mayoría lo han vivido. Un participante expresa así sus recuerdos de la etapa escolar:

"La presión, el sentirte observado en clase, el ser acusado injustamente, el que no te crean cuando dices la verdad, el tener menos credibilidad por tu condición, el rechazo directo e indirecto de los que te rodean y aunque haces todos los esfuerzos habidos y por haber para ser aceptado, al final hagas lo que hagas siempre te tratan mal" (Richy).

Sin embargo, el racismo, no ha sido un factor determinante para continuar estudiando. De hecho, haberlo sentido no implicó abandonar la trayectoria académica para las personas participantes, aunque supuso un obstáculo a salvar.

Otro factor que entorpece el seguimiento de las clases son los (2) prejuicios y los mensajes negativos que se vierten sobre el pueblo gitano, desde el desconocimiento o realizados con mala intención, por parte de payos: profesorado y/o alumnado y/o sus familias. 
"Hubo un profesor de historia que durante dos años estuvo haciendo comentarios denunciables en clase, sentando "verdades" sobre mi cultura y entrando en debates con mi hermano y conmigo intentando demostrar que la cultura "paya" era mejor que la nuestra. En una ocasión llegó a decir que los gitanos asesinaban a las mujeres que no llegaban vírgenes al matrimonio en clase, donde los alumnos creen que los profesores lo saben todo" (Brian).

Esto hace sentir al pueblo gitano que están en una escuela paya que desconoce su cultura y la infravalora, pese a su enorme riqueza y contribución a la pluralidad del país.

En línea con esto, señalan que se da una (3) total ausencia de su cultura en la escuela, algo que demandan:

"Que se nos reconozca, que se nos reconozca como pueblo, como minoría étnica en España, que se vele por la preservación de nuestra cultura como patrimonio de España, que se reconozca nuestra historia y méritos, que se reconozca nuestra lengua y se hagan cosas por compensar la pérdida de ella, que se haga una educación para nosotros también y que nos incluyan los libros e historia de España en general" (Brian).

Este aspecto contribuiría en buena medida a que la población gitana mirase a la escuela con otros ojos.

$\mathrm{Si}$ algo resulta especialmente frustrante para quien desea estudiar es el método habitual de estudio que la mayor parte han vivido en la Educación Primaria y Secundaria: (4) memorizar. Les resulta tan tedioso, inútil y duro de hacer sin apoyos, que la mayoría opta por trabajar:

"Hablo con mis primos sobre lo ridículo que es el sistema educativo, en el sentido de que lo que nos enseñan es a memorizar y a resolver problemas matemáticos de manera mecánica y no entendiéndolo realmente. No se enseña a pensar por uno mismo, y como resultado, cuando algo del problema de matemáticas cambia o te lo expresa de manera distinta la gente no sabe qué hacer" (Miguel).

Si la escuela fuera más atractiva en este sentido, todos aseguran que tendrían una mayor motivación intrínseca por el estudio.

Asimismo, (5) la falta de apoyos escolares es una debilidad. Si muchas personas gitanas contasen con recursos y apoyos fuera de la escuela la 


\section{Hernández \& Álvarez. - Trabajo y educación. Gitanos Cantabria}

experiencia escolar podría ser más satisfactoria. El participante más desmotivado de los participantes en este estudio, que sopesa abandonar sus estudios, lo ha expresado así: "El único has sido tú, el único que me ha ayudado y motivado para trabajar" (Abel). Haber tenido el apoyo de un mediador escolar gitano que le ha ayudado a hacer sus deberes ha sido estimulante para este chico. Sin embargo, estos apoyos suelen ser escasos y puntuales. Si no cuentan en su casa con personas a las que pedir ayuda, libros, etc. es difícil que logren realizar sus tareas escolares diarias.

También los participantes señalan que (6) los mensajes de desánimo de parte del pueblo gitano son negativos y la falta de expectativas de la comunidad gitana en el sistema educativo dificulta el interés en los niños y niñas. "Siempre nos advierten de la gran lucha que enfrentaremos y los más pesimistas nos dicen que saldremos muy heridos y que al final no alcanzaremos nada" (Richy). Estos mensajes de miedo pueden ser muy influyentes para las niñas y niños gitanos.

Asimismo, algunas (7) prácticas educativas empleadas con fines exclusores reciben unas pésimas valoraciones, entre las que destacan: las bajas expectativas del profesorado en el pueblo gitano, el paternalismo, hacer agrupamientos por nivel, aislar a todas las personas gitanas en un grupo de Diversificación Curricular o Educación Compensatoria, etc. "Las bajas expectativas puestas en nosotros y la adaptación curricular no solo frustraron nuestros ánimos, sino nuestra carrera escolar" (Manuel). Reivindican una educación inclusiva, sin fisuras.

En línea con este factor, también se ha señalado otra dificultad: (8) la tradicional falta de constancia y la visión cortoplacista del pueblo gitano.

"El problema de los gitanos es que seguimos pensando como nómadas, en el día a día, en que nuestro único recurso sea nuestra astucia, y jamás planificando a largo plazo y hay que cambiar eso. Hay que enseñar a invertir a largo plazo para obtener mejores resultados y más eficaces, porque lo contrario es pan pa' hoy y hambre pa' mañana" (Brian).

Parece necesario un cambio de mentalidad en el pueblo gitano al respecto.

Otro factor que aparece en las historias de vida es el (9) temor personal al fracaso y la valoración que éste produzca en la comunidad gitana: esforzarse en estudiar y no lograrlo o lograrlo y después no conseguir trabajo de lo que 
has estudiado. "También asusta el fracaso, porque los gitanos nos hinchamos para contar logros, pero rápidamente buscamos ejemplos para tirar a los demás. El tener una carrera y fracasar puede ser sinónimo de habladurías que partan tu orgullo" (Jhony). Se trata de un asunto delicado en el contexto de una familia gitana y de la comunidad gitana y que puede afectar en muy diferente grado a las personas afectadas.

Para algunas personas gitanas, estudiar es sinónimo de (10) soledad. Sienten que serán los únicos que lo hagan, los primeros en romper con la tradición y saben que de algún modo se sentirán solos.

"Lo que menos me motiva, sobre todo, es la soledad. Mi círculo de amigos, con los que me he criado toda mi vida, todos están haciendo lo que se espera de ellos y yo tengo que ser el que rompa con esa tradición. Es algo que nadie quiere. Lo que todos quieren es ser uno más y, por ello, el quedarse solo y no tener a nadie alrededor es algo que asusta" (Jhony).

Por otro lado, hay (11) rechazo laboral y algunos que han buscado trabajo en ámbitos no tradicionales para el pueblo gitano se han encontrado trabas.

"Voy al mercadillo porque no tengo donde trabajar. He perdido la cuenta de los curriculums presentados en empresas, almacenes y supermercados. Cuando presentas tu currículum y ven tu apellido gitano o tu foto te dicen "ya te llamaremos" o "el puesto ya está ocupado" o "puedes dejarlo, pero no necesitamos a nadie" y pasados unos días ves cómo la oferta de trabajo sigue ahí. Es frustrante que no te den la oportunidad por el mero hecho de ser gitano" (Richy).

Sin una adecuada sensibilización social será difícil su incorporación laboral en otros campos.

Por último, han señalado que (12) formar una familia desde jóvenes, tener hijos pronto o compaginar estudiar y trabajar dificulta seguir los estudios. "Mi vida familiar ahora no me impide o dificulta estudiar, pero pronto sí, estoy prometido y eso me dificultará un poco, nada fuera de lo normal" (Álex).

Hasta aquí hemos recogido doce factores que dificultan la continuidad educativa. Ahora recogeremos otros doce factores que favorecen la continuidad educativa. 


\section{Hernández \& Álvarez. - Trabajo y educación. Gitanos Cantabria}

\section{Factores que favorecen la continuidad educativa.}

Los factores que favorecen la continuidad educativa están muy ligados a la superación de los factores que entorpecen la misma y hemos recogido en el apartado anterior, como se muestra a continuación.

El factor que más destacan los participantes en este estudio como favorecedor de la continuidad educativa ha sido su (1) determinación personal a estudiar. Todos los participantes han manifestado tener sueños educativos fuertes que les han motivado a continuar. "En mi caso, obviamente, quiero seguir estudiando por que la educación es libertad, la educación es la capacidad de realizar aquello que quieres y te da opciones de ver otras cosas que si no has estudiado no podrías hacerlo" (Belén). Este ideal ha guiado a los participantes a continuar su trayectoria educativa y luchar contra todos los factores que les han dificultado o entorpecido su continuidad educativa. Potenciar y apoyar la determinación personal a estudiar lo que uno quiera puede ser clave.

Otro factor que favorece la continuidad educativa es el (2) orgullo de perseguir con ahínco y lograr una meta profesional.

"Suelo hablar de mi experiencia escolar ya que me siento orgulloso de conseguir la meta que me propuse, suelo contar lo interesante que puede llegar a ser lo que hago, porque realmente es muy interesante. La única razón que me movió a estudiar fue que, mayormente, un gitano no suele conseguir sus metas profesionales" (Adrián).

El deseo y la convicción de querer dedicarse a algo y ser un buen profesional en ese campo ha sido fundamental para propiciar la continuidad educativa de algunos de los participantes.

El (3) actual mercado laboral está sirviendo de acicate a muchos jóvenes a seguir con su trayectoria formativa. Ven que los puestos de trabajo tradicionales que han desempeñado corren peligro y desean vivir bien: tener estabilidad laboral y ganar un buen sueldo les motiva a superarse.

"Mi razón para estudiar es que quiero un futuro laboral digno, poder ascender tanto en sueldo como en categoría laboral, tener un buen sueldo, una vida mejor, ser más culto, ser mejor persona, aspirar a una vida mejor. Suelo hablarlo con otros gitanos, pero al ser tan pocos lo que estamos estudiando no 
es algo que puedo hacerlo mucho, pero mis amigos que no han estudiado me animan a que siga, que el mercadico se acaba y me busque la vida por otro lado" (Álex).

Un factor relevante, aunque no imprescindible, es el (4) apoyo de los padres a que sus hijos estudien.

"En mi casa, siempre, desde pequeños, han tenido las cosas claras. Han pasado fatigas, como decimos nosotros, por no tener recursos suficientes. Saben lo que cuesta todo en la vida y saben que el que quiere algo tiene que invertir tiempo y esfuerzo y, sobre todo, que hay que ser ambicioso y mirar a largo plazo" (Brian).

Ha habido jóvenes que no han contado con este apoyo y han llegado a la universidad, pero para la mayoría de la población gitana es un factor estimulante.

Otro factor destacado que ha favorecido el interés de niñas y niños gitanos por el ámbito escolar ha sido cuando han visto (5) escucha, interés, reconocimiento o respeto por su cultura.

"Hubo un momento en que llegamos a una parte del temario en el que se trataban culturas o algo parecido y le propuse al profesor que nos permitiera hablar a nosotros mismos de nuestra cultura en clase y mis compañeros quedaron encantados, después nos pidieron más” (Brian).

La visibilización de su cultura en la escuela les hace sentirse parte de la misma.

Asimismo, cuando han sentido el (6) reconocimiento de su potencial como alumnos, cuando el profesorado les ha elogiado/premiado por sus éxitos, etc. muchos de ellos han sentido que podían triunfar en la escuela.

"Contaré un hecho que a mí me emocionó mucho. Fue una vez en la asignatura de música: en un examen que la mayoría suspendió yo saqué matrícula de honor y el profesor decidió darme un premio y fue irme con otros compañeros de Bachiller a ver en el auditorio una ópera de Madanme Bobary y fue la primera vez que pude ir al palacio donde se hacen estas cosas, y más siendo gratuito. Fue muy emocionante" (Belén). 
Otro factor relevante a plantearse para favorecer la continuidad educativa está en (7) revertir las prácticas discriminadoras por prácticas más inclusivas. Un participante plantea:

"Involucrar a sus padres un poco más con el colegio para que se sientan parte de él y no lo vean como algo de fuera. No juntar a más de tres gitanos en una misma aula, y siendo así ponerlos bien separados. Premiarlos por sus buenas conductas y darles una asignatura con la que se sientan identificados. Hay que tener en cuenta que la historia del pueblo gitano, así como su cultura, es patrimonio nacional" (Jhony).

Algo que también les anima a continuar con su trayectoria educativa es saber del (8) éxito de otras personas gitanas en el sistema educativo, con especial interés en los universitarios. Todas las personas entrevistadas afirmaron conocer a gitanos en su entorno próximo que han estudiado y no solo a gitanos que fracasaron.

"No conocí a ningún gitano universitario hasta que llegué a primero de carrera. Cuando llegué tuve la suerte o la dicha de conocer a un joven gitano que estaba en un grupo de Madrid de Gitanos Universitarios, donde se reunían tres veces al año en unos encuentros estatales y allí fue donde, por primera vez en mi vida, conocí a gitanas y gitanos, con más o menos mi edad, estudiando en la universidad y que estaban haciendo una carrera universitaria, incluso algunos la habían acabado. Eso me hizo un cambio de mentalidad en mi persona y me sentí muy bien al ver que no estoy sola en el mundo [ríe]. Por eso, visibilizar a los referentes de cada ciudad para que otros niños y niñas vean que es posible y que no están solos. Añadir que los gitanos que estudian en mi familia son casi todos a raíz de mi ejemplo. Mis primos están en la Universidad, mi hermana ha vuelto a los estudios" (Belén).

Todos los (9) recursos y apoyos externos recibidos son muy bien valorados. Tener libros de texto, disponer de material escolar, recibir clases de apoyo con personas gitanas por las tardes, contar con mediadores gitanos en los centros cuando se dan conflictos, etc. son algunas de las medidas que más valoran. "Gracias a mi madre y a la asociación gitana he podido tener materiales y libros para estudiar. Eso me animó y ayudó a estudiar" (Miguel). 
Algo muy valorado también es la (10) gratuidad de la formación. "Estudiar es un lujo, y de los caros, no solo económico, también de tiempo que requiere mi familia y no se lo doy. Tengo mujer, hijos, responsabilidades, hay que trabajar, pagar..." (Roberto). Están agradecidos por la buena actitud de quienes han contribuido a facilitar económicamente que pudieran estudiar. "Recuerdo que mi madre me compraba "de fiado" los libros y todo el material escolar en Librería Elle y lo pagaba poco a poco durante meses" (Richy). La educación supone una inversión de esfuerzo, tiempo y dinero que muchas familias gitanas que disponen de pocos ingresos no pueden permitirse.

Por otro lado, otro factor que favorece que una persona gitana continúe su trayectoria formativa es el (11) éxito en el logro de aprendizajes cuando uno autónomamente se lo propone. Las personas participantes en este estudio han podido relatar experiencias exitosas aprendiendo autodidactamente, ya sea por iniciativa propia, dentro o fuera del sistema educativo. "Hice cursillos de todo tipo: Informática, primeros auxilios, riesgos laborales, programación...por placer. Y leí libros de Cultura Greco-Romana" (Richy).

Otro factor relevante que puede influir en la continuidad educativa del pueblo gitano es el (12) arrepentimiento que tienen y manifiestan permanentemente los que ya son adultos por no haber estudiado cuando tuvieron la oportunidad y que les hace replantearse su tarea como padres de una nueva generación. Todos los participantes en este estudio manifiestan, sin dudar, con gran ilusión, que esperan que sus hijos estudien.

"Mi generación no nos lo tomamos en serio y no estudiamos mucho. No fuimos mucho a la escuela, aunque ahora me arrepiento. Siempre me llamó la atención la carrera de derecho, me casé joven, tengo hijos, casa, negocios, responsabilidad, de joven no lo valoré, pero tenía que haberlo hecho. Que mis hijos estudien va a ser mi prioridad" (Moisés).

En línea con esto, se observa que los participantes en este estudio que ya son padres valoran mucho la escuela y las actividades que desde ella se promueven. 
"Mis hijos actualmente acuden a clases de artes marciales, inglés, baloncesto, piano y teatro. Sus notas académicas son buenas y luchamos para que cumplan su sueño y el mío, a pesar del rechazo y las dificultades diarias que ellos enfrentan cada día en el colegio" (Richy).

\section{Conclusiones}

Este estudio se suma a la reciente línea de investigación sobre la continuidad educativa del pueblo gitano. Los relatos biográficos realizados han permitido identificar doce factores que la comunidad gitana cántabra considera que dificultan la continuidad educativa de la población gitana, así como doce factores facilitadores. Algunos de los mismos coinciden con los resultados aportados por los estudios previos, como se desgranarán detenidamente a continuación.

Entre los factores que dificultan la continuidad educativa de la población gitana sigue teniendo un gran peso el racismo, dándose una total ausencia de su cultura en la escuela (Bitrián Ferrer \& Agost Felip, 2018; Díez-Palomar et al., 2018; Marques Gonçalves, 2020; Rostas \& Kostka, 2014) pese a la riqueza cultural del pueblo gitano (Carmona Santiago et al., 2019; Lorenzo Moledo et al., 2020). Cuando se aborda la cultura gitana es frecuente que aparezcan numerosos prejuicios y mensajes negativos (Abajo \& Carrasco, 2004; Dimitrova, Ferrer-Wreder, et al., 2018). Estas dificultades requieren acciones de formación, sensibilización y difusión del patrimonio cultural gitano en los centros educativos y a nivel social, ya que también las empresas tienden a poner trabas a la contratación de personas gitanas incluso cuando están cualificadas (Mihaela \& Luminița, 2020).

Otros factores que dificultan la continuidad educativa de la población gitana guardan relación con limitaciones de las instituciones educativas: el predominio de unas enseñanzas académicas siguiendo formas memorísticas o las prácticas educativas empleadas con fines exclusores (Rostas \& Kostka, 2014). Se necesita una mayor formación y sensibilización al respecto y la experiencia croata puede ser un ejemplo a seguir (Curcic et al., 2018). Asimismo, por otro lado, es precisa una intervención alentadora con niñas y niños gitanos para prevenir la aparición de sentimientos y pensamientos negativos sobre la educación. Es preciso que tengan referentes de otras personas que estudian para evitar en los que lo hagan un sentimiento de soledad o temor al fracaso, como refiere la experiencia búlgara, ya que tan 
importante es tener recursos como atender las dimensiones actitudinales (Dimitrova, Ferrer-Wreder, et al., 2018). Asimismo, es importante que cuenten con apoyos educativos, a ser posible para hacer deberes escolares y procedentes de su comunidad próxima (Aguilar Jurado et al., 2020), ya que a los estudiantes les motiva a estudiar ver a profesorado gitano.

Otros factores que dificultan la continuidad educativa de la población gitana requieren una implicación de la comunidad gitana y un cambio en sus tradiciones: la habitual falta de constancia y la visión cortoplacista del pueblo gitano, la tradicional falta de expectativas de la comunidad gitana en el sistema educativo o la tendencia a formar una familia desde jóvenes, tener hijos pronto dificultan la continuidad educativa. Es importante que las asociaciones que trabajan con el pueblo gitano actúen en esta dirección para contribuir a que florezcan los sueños académicos y profesionales de niñas, niños y jóvenes gitanos. En la medida que se puedan revertir todos estos factores que dificultan la continuidad educativa y promover los que la facilitan, más fácil será lograr una mayor inclusión e igualdad de oportunidades.

Como afirma un estudio previo, las trayectorias educativas no están predeterminadas, sino que son moldeadas por los participantes en sus interacciones con la escuela, la familia y las redes sociales más amplias (Alexiadou, 2019). Un aporte relevante de este trabajo ha sido identificar que el factor más relevante en la continuidad educativa de la población gitana reside en la determinación personal a estudiar del propio sujeto, algo que en un estudio previo aparecía tan claramente, ocupando un tercer lugar (Abajo \& Carrasco, 2004). Esto significa, entre otras cosas, que en el pueblo gitano hay personas que desean estudiar (sin sentirse menos gitanas por ello). Es necesario evidenciar esta realidad y lograr apoyar a estas personas en el logro de su objetivo para que el resto de factores no termine por ahogar los sueños académicos de las personas gitanas. Todos los participantes en este estudio son conscientes de su éxito en el logro de aprendizajes cuando autónomamente se lo han propuesto.

Resulta muy satisfactorio para las personas gitanas perseguir con ahínco y lograr una meta profesional y este éxito es importante que sea celebrado por la comunidad como una conquista de muchas más que se espera que vengan. El actual mercado laboral, fruto de la actual crisis propiciada por la pandemia de COVID-19, puede contribuir a que muchas personas se formen 


\section{Hernández \& Álvarez. - Trabajo y educación. Gitanos Cantabria}

para optar a trabajos diferentes de los tradicionales que requieren titulación. Aún son pocos los universitarios en Cantabria, pero el arrepentimiento que tienen y manifiestan permanentemente los que ya son adultos por no haber estudiado cuando tuvieron la oportunidad les ha hecho replantearse seriamente su tarea como padres de una nueva generación y es muy posible que las niñas y niños gitanos de hoy tengan muchas más oportunidades, apoyos y facilidades de las que tuvieron sus padres en el pasado. En esta línea en las familias es muy recomendable el apoyo de los padres y familiares a que sus hijos estudien. En sintonía, es preciso demandar en los centros que conozca, reconozca y respete la cultura gitana, que se reconozca el potencial del alumnado gitano y que se reviertan las prácticas discriminadoras por prácticas más inclusivas estudien (Abajo \& Carrasco, 2004; Díez-Palomar et al., 2018). Igual de importante es visibilizar el éxito de las personas gitanas en el sistema educativo que han concluido sus estudios, con especial interés en los universitarios en tanto que se contribuye a que el pueblo gitano tenga referentes de personas gitanas y formadas.

Por último, pero no por ello es menos importante, se necesita que haya prácticas, recursos y políticas de bienestar en torno a la igualdad y las oportunidades (Abajo \& Carrasco, 2004; Alexiadou, 2019; Díez-Palomar et al., 2018),ya que los recursos y apoyos externos recibidos son muy bien valorados y las tasas que tienen algunas matrículas complican la apuesta educativa de numerosas familias gitanas sin programas de becas (Abajo \& Carrasco, 2004).

Se han producido avances en Cantabria dado que empieza a haber gitanos con estudios universitarios, y aunque es posible que haya retrocesos (los jóvenes escolarizados ahora en la ESO que están teniendo éxito y podrían cursar estudios universitarios son pocos), este estudio da pie a pensar que la comunidad gitana está atravesando una fase de tránsito entre tradición y modernidad y que hay una alta probabilidad de que las nuevas generaciones apuesten por la continuidad educativa, al observarse algunos cambios significativos: primeras generaciones de adultos/as que se sacan la ESO, gitanos/as jóvenes que se animan a estudiar ciclos formativos de grado medio y superior, primeros estudiantes universitarios, etc. así como padres/madres gitanos/as jóvenes que se implican con lo escolar, dibujándose una tímida nueva tendencia educativa de mayor cooperación en pro del éxito escolar. 


\section{Referencias}

Abajo, E., \& Carrasco, S. (2004). Experiencias y trayectorias de éxito escolar de gitanas y gitanos en España. Encrucijadas sobre educación, género y cambio cultural. CIDE / Instituto de la Mujer.

Aguilar Jurado, M. Á., Gil Madrona, P., \& Ortega Dato, J. F. (2020). Efectos del programa Promociona contra el fracaso escolar en alumnos de raza gitana. Revista de Investigación Educativa, 38(2), 345-358.

https://doi.org/10.6018/rie.396131

Alexiadou, N. (2019). Framing education policies and transitions of Roma students in Europe. Comparative Education, 55(3), 422-442. https://doi.org/10.1080/03050068.2019.1619334

Baracsi, K. (2016). the Unmaking of Roma Students: Contributing From a Critical and Engaged Ethnography. The Age of Human Rights Journal, 7(7), 77. https://doi.org/10.17561/tahrj.n7.5

Bitrián Ferrer, L., \& Agost Felip, M. R. (2018). Experiencia, percepción y expectativas de adolescentes absentistas gitanos. Àgora De Salut, 5, 227-234. https://doi.org/10.6035/agorasalut.2018.5.25

Cantón-Delgado, M. (2018). Narratives of the Gypsy awakening. Religious innovation, Gypsy leadership and identity politics. Revista

Internacional de Sociologia, 76(2).

https://doi.org/10.3989/ris.2018.76.2.16.96

Carmona Santiago, J., García, M., Máiquez, M. L., \& Rodrigo, M.-J. (2019). El impacto de las relaciones entre la familia y la escuela en la inclusión educativa de alumnos de etnia gitana. Una revisión sistemática. Multidisciplinary Journal of Educational Research, 9(3), 319. https://doi.org/10.17583/remie.2019.4666

Curcic, S., Lapat, G., Susec, B., \& Ignac, B. (2018). From past aspirations to present achievements: A case study of three K-8 schools successfully educating Roma students. International Journal of Multicultural Education, 20(2), 24-41. https://doi.org/10.18251/ijme.v20i2.1638

Díez-Palomar, J., de Sanmamed, A. F. F., García-Carrión, R., \& MolinaRoldán, S. (2018). Pathways to equitable and sustainable education through the inclusion of Roma students in learning mathematics.

Sustainability (Switzerland), 10(7). https://doi.org/10.3390/su10072191 Dimitrova, R., Ferrer-Wreder, L., \& Ahlen, J. (2018). School Climate, Academic Achievement and Educational Aspirations in Roma Minority 
44 Hernández \& Álvarez. - Trabajo y educación. Gitanos Cantabria

and Bulgarian Majority Adolescents. Child and Youth Care Forum, 47(5), 645-658. https://doi.org/10.1007/s10566-018-9451-4

Dimitrova, R., Johnson, D. J., \& van de Vijver, F. J. R. (2018). Ethnic

socialization, ethnic identity, life satisfaction and school achievement

of Roma ethnic minority youth. Journal of Adolescence, 62, 175-183.

https://doi.org/10.1016/j.adolescence.2017.06.003

Goenechea, C., \& Gallego, B. (2020). Voces del alumnado gitano sobre su

experiencia en la universidad. Profesorado, 24(2), 462-482.

https://doi.org/10.30827/profesorado.v24i2.15157

Helakorpi, J., Lappalainen, S., \& Sahlström, F. (2019). Becoming tolerable:

subject constitution of Roma mediators in Finnish schools.

Intercultural Education, 30(1), 51-67.

https://doi.org/10.1080/14675986.2018.1537671

Lorenzo Moledo, M., Míguez Salina, G., \& Cernadas Ríos, F. X. (2020).

Can funds of knowledge contribute to the involvement of Roma families in schools? Basis for an educational project. Teoria de La

Educacion, 32(1), 191-211. https://doi.org/10.14201/teri.21299

Marques Gonçalves, G. (2020). Experiencing everyday racism : narratives of Antigypsyism in Spain. International Journal of Roma Studies, 2, 6686. https://doi.org/10.17583/ijrs.2020.6072

Mihaela, R., \& Luminița, S. M. (2020). Social perception of the integration of Romani people ( Gypsies ) into the labor market. Technium Social Sciences Journal, 5, 170-184.

O'Nions, H. (2015). Narratives of social inclusion in the context of roma school segregation. Social Inclusion, 3(5), 103-114.

https://doi.org/10.17645/si.v3i5.258

Rostas, I., \& Kostka, J. (2014). Structural dimensions of Roma school desegregation policies in central and Eastern Europe. European Educational Research Journal, 13(3), 268-281. https://doi.org/10.2304/eerj.2014.13.3.268

Vargas, J., Davila Legerén, A., \& Flecha García, R. (2004). Metodología comunicativa crítica en la investigación en ciencias sociales: la investigación WORKALÓ. Lan Harremanak, 2(11), 21-34. 
Carlos Hernández-Borja es un mediador gitano de la Fundación Diagrama en Cantabria (España)

Carmen Álvarez-Álvarez es profesora contratada doctora del Departamento de Educación de la Universidad de Cantabria (España)

Contact address: carmen.alvarez@unican.es 